\section{Qualidade de vida e medidas de utilidade: parâmetros clínicos para as tomadas de decisão em saúde}

\author{
Alessandro Gonçalves \\ Campolina ${ }^{1} e$ \\ Rozana Mesquita Ciconelli²
}

Palavras-chave: Custos e análise de custos, satisfação do paciente, qualidade de vida, técnicas de apoio para a decisão.

Universidade Federal de São Paulo (UNIFESP), Departamento de Medicina, Disciplina de Reumatologia. Enviar correspondência para este autor no seguinte endereço: Avenida Dr. Altino Arantes 928/23, Mirandópolis, CEP 04042-004, São Paulo, SP, Brasil. E-mail: alecampolina@yahoo.com.br

2 UNIFESP, Departamento de Medicina, Disciplina de Reumatologia. E-mail: rciconelli@reumato.epm.br
Nas últimas décadas, o interesse crescente da comunidade científica e de todo o setor saúde em questões de qualidade de vida levou a um desenvolvimento marcante desse constructo (1). Com o propósito de prover maior acurácia às avaliações individuais e coletivas dos estados de saúde, um grande número de instrumentos tem sido produzido e validado em todo o mundo $(1,2)$. As medidas de qualidade de vida têm aplicações diversas: triagem e monitoramento de problemas psicossociais no cuidado individual, estudos populacionais sobre percepção de estados de saúde, auditoria médica, medidas de resultados em serviços de saúde, ensaios clínicos e análises econômicas que enfocam o custo monetário necessário para garantir uma melhor qualidade de vida (custo-utilidade) (2).

Em grande parte impulsionadas pelos avanços tecnológicos na área médica, as pesquisas no campo da qualidade de vida têm levado clínicos, cientistas, economistas e gestores a se interessarem pelos impactos trazidos por essas tecnologias em termos de estados de saúde e custos para os sistemas de saúde (3). Por um lado, todo esse avanço tem levado à prevenção e ao diagnóstico precoce de muitas doenças, além de possibilitar um tratamento mais efetivo para muitas doenças que cursam com uma sobrevida pequena, levando a um tempo de vida maior (4). Por outro lado, os custos dos sistemas de saúde tornam-se cada vez maiores, mesmo nos países industrializados, especialmente à medida que aumenta a fração muito velha da população idosa, ou seja, o grupo de indivíduos acima de 80 anos. Ao invés de processos agudos que se "resolvem" rapidamente através da cura ou do óbito, tornam-se predominantes as doenças crônicas e suas complicações, que implicam em décadas de utilização dos serviços de saúde (5).

Em face dessa mudança no perfil de morbimortalidade, com um aumento na prevalência das doenças crônicas e de suas seqüelas, os próprios objetivos da atenção à saúde passaram a ser questionados, sendo que cada vez mais esses objetivos têm deixado de ser simplesmente a "cura" e passado a ser a "melhoria da vida" dos pacientes $(6,7)$.

Dentro desse contexto, as medidas que permitem a tomada de decisão com base nas preferências por estados de saúde e a realização de análises econômicas focadas na perspectiva do paciente - as medidas de utilidade - elevam a qualidade de vida ao patamar de princípio essencial para os sistemas de saúde no novo milênio (8). O objetivo 
desse trabalho é apresentar e contextualizar esses conceitos no setor saúde.

\section{QUALIDADE DE VIDA}

Foi particularmente na década de 1960 que o constructo qualidade de vida passou a ser entendido como qualidade de vida subjetiva ou qualidade de vida percebida pelas pessoas. Em grande parte influenciado pela Organização Mundial da Saúde (OMS), que declara que a saúde não se restringe à ausência de doença, mas engloba a percepção individual de um completo bem-estar físico, mental e social, o conceito ampliou-se para além da significação do crescimento econômico, buscando envolver os diversos aspectos do desenvolvimento social (9). O que existe atualmente é um conceito ainda amorfo, não consensual, com múltiplas aplicações nas mais diversas disciplinas e campos do conhecimento humano. Apesar disso, o que de fato caracteriza o conceito de qualidade de vida é a subjetividade e a multidimensionalidade (10).

De uma maneira geral, a percepção individual do estado de saúde (subjetividade) tem sido avaliada em grandes domínios ou dimensões da vida (2). Em termos práticos, os domínios são conjuntos de questões agrupadas nos instrumentos de avaliação e que se referem a uma determinada área do comportamento ou da condição humana, por exemplo: domínio psicológico, econômico, espiritual, social, físico, etc.

Além disso, duas abordagens principais têm sido utilizadas para a avaliação da qualidade de vida relacionada à saúde: as medidas psicométricas (das quais não trataremos neste trabalho) e as medidas de preferências por estados de saúde (11). A literatura científica classifica os instrumentos de avaliação de qualidade de vida como genéricos e específicos $(12,13)$.

Os instrumentos genéricos têm como vantagens a possibilidade de avaliação simultânea de várias áreas ou domínios, a possibilidade de serem usados em qualquer população e o fato de permitirem comparações entre pacientes com diferentes patologias. A grande desvantagem é o fato de que podem não demonstrar alterações em aspectos específicos. Existem basicamente dois tipos: os perfis de saúde $(12,14)$, como o Short-Form Health Survey (SF-36), o Nottingham Health Survey (NHP), o Sickness Impact Profile (SIP), o McMaster Health Index Questionnaire (MHQ), e os índices de saúde ou medidas de utilidade, que refletem a preferência dos pacientes por um determinado estado de saúde ou por um determinado tratamento, relacionando em escalas quantitativas diversos cenários possíveis e variáveis, desde a saúde perfeita até a morte (15).
Os instrumentos específicos de avaliação da qualidade de vida são destinados a determinadas doenças (12) (diabetes, doença pulmonar obstrutiva crônica e artrite, por exemplo), a determinadas populações (crianças, adultos, idosos) ou a determinadas funções (capacidade funcional ou função sexual, por exemplo). A grande vantagem é o fato de serem clinicamente mais sensíveis; porém, não permitem comparações entre patologias distintas e são restritos aos domínios de relevância do aspecto a ser avaliado.

\section{MEDIDAS DE UTILIDADE}

O conceito de utilidade, como empregado aqui, deriva da teoria econômica de tomada de decisão sob incerteza, publicada em 1944 por John von Neumann e Oscar Morgenstern (16). O princípio fundamental desse paradigma normativo é que os indivíduos têm preferências e tomam decisões diante da incerteza. Portanto, o termo utilidade, neste contexto, não tem relação com "serventia" (como é empregado no cotidiano), nem com utilitarismo (como é empregado por algumas escolas filosóficas) (16). Na verdade, dentro do paradigma descrito por von Neumann-Morgenstern, que tem sido dominante nas últimas quatro décadas, a utilidade reflete as preferências diante da incerteza, o que, no caso da saúde, vem a ser as preferências por determinados estados de saúde (12).

Como veremos adiante, a grande vantagem de se trabalhar com avaliações de qualidade que se baseiam em medidas de utilidade é que elas permitem a estruturação de árvores de decisão, um elemento fundamental das análises de decisão, assim como o cálculo de anos de vida ajustados pela qualidade (quality-adjusted life years, QALYs), um elemento fundamental das análises econômicas do tipo custo-utilidade.

\section{Tipos de preferências}

Como nem sempre as terminologias são aplicadas de forma consistente nessa área de conhecimento, é fundamental diferenciar preferência, utilidade e valor (17). Preferência é o conceito mais amplo, que compreende os conceitos de utilidade e de valor. Utilidade é um tipo específico de preferência, medido sob condições de incerteza, de acordo com o paradigma fundado por von NeumannMorgenstern. Valor é uma preferência medida sob condições de certeza, de modo a não expressar a atitude subjetiva de risco (18).

As preferências podem ser de dois tipos: ordinais e cardinais (19). As preferências ordinais são 
aquelas que apenas ordenam os estados alternativos, do mais preferido para o menos preferido $(A>B>C)$. As preferências cardinais são medidas intervalares, onde os intervalos, e não as razões, apresentam significado, por exemplo: numa escala intervalar onde $\mathrm{A}=0,60, \mathrm{~B}=0,30$ e $\mathrm{C}=0,15$, é inapropriado dizer que $A$ é duas vezes mais preferido que $B$, mas é apropriado dizer que a diferença entre $\mathrm{A}$ e $\mathrm{B}$ é duas vezes maior que a diferença entre $B$ e $C$ (19).

Por fim, quando falamos em utilidades, estamos falando em preferências cardinais, medidas em condição de incerteza.

\section{Medidas diretas}

Existem basicamente três técnicas bem estabelecidas para a medida direta (ou seja, que questiona o indivíduo diretamente sobre sua preferência) de utilidades: a escolha pela chance (standard gamble), a escolha pelo tempo (time trade-off) e a escala visual analógica (20).

$\mathrm{Na}$ técnica de escolha pela chance, solicita-se aos respondentes que escolham entre duas alternativas. Em uma alternativa é oferecido um estado de saúde intermediário (por exemplo, uma dor lombar crônica), sob condição de certeza. Em outra alternativa, são oferecidos dois estados de saúde sob condição de incerteza caso o paciente se submeta a um procedimento terapêutico: um estado de saúde que seria o melhor possível (com probabilidade $P$ de ocorrer) e um que seria o pior possível, como, por exemplo, a morte (com probabilidade $1-P$ ). Com base nas respostas dadas, a probabilidade $P$ é variada até que se determine o ponto de indiferença $P^{*}$ - ponto no qual o indivíduo ficaria indiferente entre abandonar uma situação de certeza (dor lombar crônica) para se submeter a um procedimento capaz de trazer resultados incertos ou permanecer na situação de certeza, sem se submeter ao procedimento terapêutico. Esse ponto de indiferença $P^{*}$ é a utilidade que o respondente atribui ao estado de saúde intermediário apresentado inicialmente, numa escala em que a utilidade do pior estado é 0,00 e a do melhor estado é 1,00 (18).

$\mathrm{Na}$ técnica de escolha pelo tempo, solicita-se aos respondentes que indiquem quantos anos de vida estão dispostos a ceder em troca de evitar um determinado estado de saúde crônico. Em uma alternativa, é oferecido ao respondente um estado de saúde crônico para o resto da vida (por exemplo, a dor lombar). Na outra alternativa, é oferecido a ele um estado de saúde melhor por um período de tempo menor (por exemplo, sem dor por 10 anos, seguido de morte imediata). $\mathrm{O}$ período de tempo menor vai sendo variado (15 anos, 20 anos . . .) até que se determine o ponto de indiferença, ou seja, o momento no qual o indivíduo se torna indiferente diante das duas alternativas (viver com a condição crônica ou perder alguns anos de vida) e portanto poderia enfrentar um possível procedimento terapêutico mesmo que isso significasse viver menos. Em termos da escala de utilidade, o valor 0,00 indica a disposição de abrir mão de todos os anos adicionais de vida em troca de uma saúde perfeita e o valor 1,00 indica a preferência por não abrir mão de nenhum ano de vida (21).

A escala visual analógica é uma linha desenhada onde uma extremidade equivale a 0 e a outra equivale a 1 . $\mathrm{O}$ valor 1 pode corresponder ao melhor estado de saúde possível e o 0 ao pior possível (por exemplo, a morte). Após marcar na linha um ponto que indique o melhor e o pior estados de saúde possíveis, o respondente marca, na mesma linha, o estado de saúde em avaliação ou o seu próprio estado de saúde. Cada ponto corresponderá, portanto, ao valor de utilidade atribuído a cada um dos estados de saúde indicados (20) (figura 1).

Todas as três técnicas são realizadas com o apoio de recursos visuais. A única técnica capaz de capturar preferências do tipo utilidade é a escolha pela chance, conhecida também como "utilidade verdadeira". As demais técnicas captam preferências do tipo valor.

\section{Medidas indiretas}

As medidas diretas de preferências, utilizando as técnicas descritas acima, são em geral complexas, demoradas e custosas (18). Uma abordagem mais prática, e que tem sido mais comumente empregada, é a utilização de sistemas multiatributos, que, através de questionários, permitem descrever e calcular preferências para diversos estados de saúde. Entre os instrumentos mais conhecidos estão: o EuroQol-5D (22), o Quality of Well-Being Scale (23), o Health Utilities Index (24) e mais recentemente o SF-6D (25-28).

Um dos sistemas mais utilizados atualmente é o Health Utilities Index Mark III (HUI Mark III), desenvolvido no Canadá e utilizado em várias pesquisas populacionais em saúde e em levantamentos estatísticos sociais naquele país. O sistema é composto de oito atributos, com cinco ou seis níveis de capacidade possíveis para cada atributo. O HUI Mark III pode descrever um total de 972000 estados únicos de saúde (24). São atributos desse sistema: visão, audição, fala, deambulação, destreza, emoção, cognição e dor. De acordo com o nível de resposta em cada atributo, é feita a descrição do estado de saúde, sendo em seguida aplicada uma "função utilidade" que permite determinar a utilidade para o estado de saúde descrito. 
FIGURA 1. Escala visual analógica para a medida direta de utilidade ${ }^{a}$

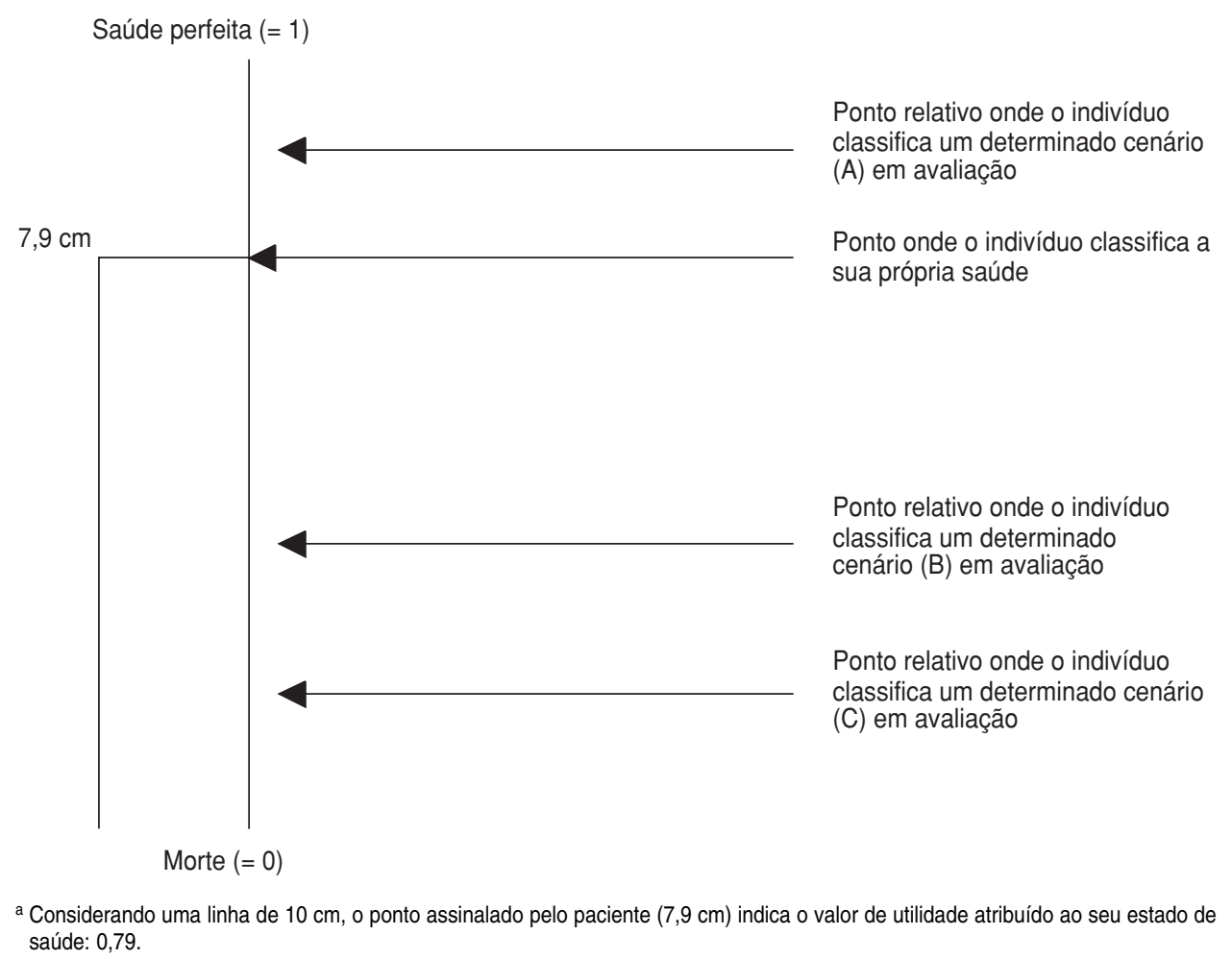

Mais recentemente, várias tentativas de cálculo de utilidades têm sido feitas a partir de dados levantados com o SF-36, um instrumento genérico de qualidade de vida amplamente utilizado em diversos ensaios clínicos (29). A partir dessas tentativas, desenvolveu-se o SF-6D, um instrumento utilizado como ponte entre o SF-36 e as medidas de utilidade, permitindo comparações com dados produzidos a partir do HUI Mark III. O SF-36, composto por seis dimensões de saúde (funcionamento físico, limitação global, funcionamento social, dor no corpo, saúde mental e vitalidade), descreve um total de 9000 estados de saúde com níveis de capacidade variando de 2 a 6 (25).

\section{ANÁLISE DE DECISÃO EM SAÚDE}

\section{O que é e para que serve?}

A análise de decisão pode ser definida como uma abordagem sistemática para a tomada de decisões em condições de incerteza (30). É uma técnica que permite aos tomadores de decisão compararem desfechos em diferentes estratégias. No campo da saúde, esse tipo de análise tem sido aplicado para avaliar diferentes estratégias diagnósticas e terapêuticas (31).

O método deriva da teoria dos jogos, desenvolvida na década de 1950 e aplicada inicialmente na produção industrial, no início dos anos 1960. Apenas mais recentemente os periódicos médicos têm trazido publicações descrevendo metodologias e aplicações na área médica, despertando o interesse de clínicos e educadores, que têm utilizado essa modalidade de análise para a resolução de problemas individuais de pacientes e de políticas públicas (32).

$\mathrm{Na}$ área terapêutica, por exemplo, podem-se comparar três diferentes abordagens para pacientes com coronariopatia: tratamento medicamentoso, angioplastia e revascularização miocárdica. $\mathrm{Na}$ área diagnóstica, podem-se comparar alternativas para a abordagem de nódulos tiroidianos, como punção aspirativa com agulha fina e técnicas não-invasivas de imagem. Na área de prevenção e promoção de saúde, podem ser comparadas, por exemplo, estratégias de triagem para câncer de próstata com estratégias expectantes (11). Em geral, o método é bem aplicado em situações clínicas, onde as decisões precisam ser tomadas sem o suporte de evidências científicas diretas e baseadas em ensaios clínicos controlados e randomizados. 
Algumas etapas são fundamentais para a utilização da técnica de análise de decisão para resolver problemas difíceis na prática clínica. Basicamente, temos cinco etapas: 1) formular uma pergunta explícita; 2) estruturar a decisão (montar a árvore de decisão); 3) preencher a árvore com dados (probabilidades e desfechos); 4) determinar o valor de cada estratégia (utilidades); e 5) realizar análises de sensibilidade (33).

A medicina baseada em evidências contribuiu bastante para esse processo, visto que as probabilidades de desfechos clínicos são extraídas basicamente de estudos, bancos de dados e opiniões de especialistas. Do mesmo modo, um método para classificar os desfechos conforme a preferência dos indivíduos que serão submetidos a intervenções também é valioso. O método mais comumente empregado na classificação de estados de saúde para fins de tomada de decisão são as já descritas medidas de utilidade (34).

Portanto, as medidas de utilidade constituem uma etapa fundamental das análises de decisão, por permitirem avaliar e comparar as preferências individuais e populacionais por desfechos possíveis. Seja como for, a essência das análises de decisão é comparar riscos e benefícios de estratégias diferentes, incorporando três componentes básicos: escolhas (estratégias alternativas), probabilidades (chances de ocorrências de cada desfecho clínico) e valores (indicadores quantitativos das preferências dos pacientes por cada um dos desfechos clínicos prováveis) (35). Para tornar explícita essa ponderação de riscos e benefícios, que muitas vezes é feita implicitamente pelo julgamento clínico, as análises de decisão utilizam um recurso prático, que é a estruturação das árvores de decisão.

\section{As árvores de decisão}

A estruturação de uma árvore de decisão começa com a lista dos possíveis desfechos. Em parte, a arte da análise de decisão consiste em reduzir uma decisão complexa a um número finito de elementos simples, o que atualmente já pode ser feito com o apoio de diversos tipos de software (35). Portanto, uma árvore de decisão deve ser complexa o suficiente para incorporar todos os elementos chaves e valores que são importantes para os pacientes e, ao mesmo tempo, simples o suficiente para ser compreensível e operacional.

O exemplo a seguir ilustra como se dá o processo de tomada de decisão a partir de uma situação clínica hipotética (figura 2). Podemos considerar uma estratégia terapêutica com três possibilidades de desfechos clínicos: morte, vida sem morbidade e vida com morbidade. Considerando que os valores (utilidade) atribuídos pelos pacientes a cada um desses estados é 0 para a morte, 1 para a vida sem morbidade e 0,5 para a vida com morbidade, e assumindo que a probabilidade de cada desfecho seria, respectivamente, 0,25,

FIGURA 2. Árvore de decisão utilizada para análise de decisões em saúde ${ }^{a}$

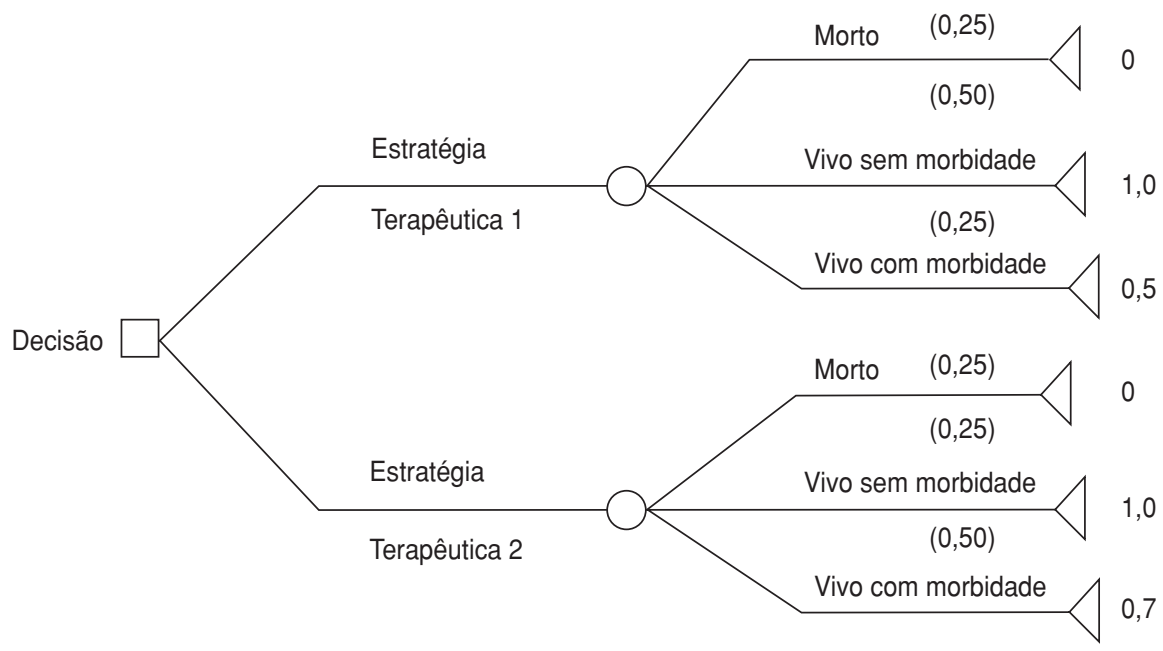

a $\mathrm{O}$ quadrado na figura indica um ponto de decisão; os círculos indicam os pontos de chance; e os triângulos indicam os desfechos quantitativos (medidos em utilidades). Os números entre parênteses indicam a probabilidade de ocorrência de cada desfecho possível. 
0,50 e 0,25 , teríamos que a média ponderada para essa estratégia terapêutica seria $0 \times 0,25+0,5 \times 0,5$ $+1,0 \times 0,25=0,5$. Essa ponderação é denominada de "valor esperado" da estratégia. Se procedermos do mesmo modo para todas as estratégias ou escolhas que compõem a árvore de decisão, poderemos determinar aquela com maior "valor esperado" e que provavelmente será a mais desejável para a resolução do problema em questão.

\section{ANÁLISE DE CUSTO-UTILIDADE}

\section{Importância das avaliações econômicas em saúde}

As avaliações econômicas destinam-se a estimar os custos de formas alternativas de assistência à saúde, comparando, por exemplo, estratégias clínicas com estratégias cirúrgicas (15).

Existem basicamente quatro tipos de análises econômicas em saúde: as análises de custo-minimização, as análises de custo-benefício, as análise de custo-efetividade e as análises de custo-utilidade (34, 36). As análises de custo-minimização simplesmente determinam qual é a intervenção mais barata, enquanto que os outros três tipos estabelecem relações. De um modo geral, a diferença básica é que as análises de custo-benefício estabelecem uma relação entre os recursos econômicos gastos e os recursos economizados; as análises de custo-efetividade estabelecem essa mesma relação entre os recursos econômicos gastos e os efeitos clínicos produzidos; e as análises de custo-utilidade estabelecem uma relação entre os recursos gastos e a qualidade de vida ganha $(37,38)$. As análises econômicas são constituídas de dois conceitos fundamentais: a perspectiva da análise e o tipo de efetividade clínica analisada (33).

A perspectiva de uma análise é o ponto de vista a partir do qual a análise é conduzida, podendo ser a perspectiva do paciente, a perspectiva de um programa (um prestador de serviço), a perspectiva do pagador (um plano de saúde) ou a perspectiva da sociedade.

A efetividade clínica deve ser expressa nas unidades mais convenientes e apropriadas à análise. Tais unidades podem ser medidas de mortalidade (anos de vida ganhos, número de vidas salvas), morbidade (complicações ou procedimentos evitados, dias de trabalho perdidos, qualidade de vida relacionada a várias condições clínicas), identificação da doença e terapia (número de casos diagnosticados, número de casos tratados apropriadamente) e a combinação de morbidade e mortalidade (que nada mais é do que os anos de vida ajustados pela qualidade, os quality-adjusted life years, ou QALYs)

Por fim, o fundamental das análises econômicas é que elas expressem ao final os custos e a "efe- tividade incremental", ou seja, as diferenças entre as duas estratégias comparadas em termos de custo e efetividade ou o custo extra por unidade de efetividade ganha (39).

A análise de custo-utilidade pode ser vista como uma análise econômica tipo custo-efetividade realizada a partir da perspectiva do paciente e que toma como parâmetro de efetividade clínica os QALYs (40). O conceito de QALYs foi desenvolvido na década de 1970 a partir de estudos sobre insuficiência renal crônica (16). A vantagem desse indicador de saúde é que ele permite simultaneamente capturar ganhos com a redução da morbidade (ganhos em qualidade) e com a redução da mortalidade (ganhos em quantidade), integrando-os em uma única medida (39). Por exemplo, uma intervenção que estendesse a vida de um indivíduo $A$ por 1 ano, em um nível de qualidade de 0,50, e que melhorasse a qualidade de vida de um indivíduo $B$ em 0,25 , por 1 ano, produziria 0,75 QALYs.

A utilização do modelo QALY tem implicações éticas e econômicas importantíssimasprimeiramente por que a medida de QALY é independente de idade, ou seja, adicionar um QALY a um indivíduo de 25 anos é o mesmo que adicionálo a um de 85 anos, visto que, em ambos os casos, estaríamos garantindo vida com qualidade. Segundo, "QALY ganho é QALY ganho": ganhar 0,50 em qualidade de vida para uma pessoa é o mesmo que ganhar 0,25 para duas pessoas; ou ganhar 0,50 em qualidade de vida por 1 ano é o mesmo que ganhar 0,25 por 2 anos (41).

O conceito de QALY ganho pode ser melhor compreendido com alguns exemplos simples (10). Podemos considerar um indivíduo cuja qualidade de vida é reduzida a 0,03 por 30 anos pelo uso de anti-hipertensivos, a fim de ganhar 10 anos de sobrevida com um nível de qualidade de 0,9 . O QALY ganho por esse indivíduo é de $10 \times 0,9-30 \times$ $0,03=8,1$. Da mesma forma, poderíamos considerar uma programa que estende a expectativa de vida de indivíduos por 2 anos em um nível de qualidade de 0,50 e que melhora a qualidade de vida de outros indivíduos de 0,50 para 0,75 por 2 anos. O QALY ganho para o grupo total de indivíduos seria de $2 \times$ $0,50+2 \times 0,25=1,5$. Tal exemplo pode ser melhor visualizado através da figura 3 .

Incorporando o conceito de QALY ganho às análises econômicas, teríamos que a fórmula básica para a avaliação de custos e conseqüências de duas ou mais intervenções (análise de custo-utilidade) seria (42):

$$
\mathrm{AE}=\frac{\text { custo } 2-\text { custo } 1}{\text { QALY ganho } 2-1}
$$


FIGURA 3. Anos ajustados pela qualidade de vida (QALYs) ganhos ${ }^{a}$

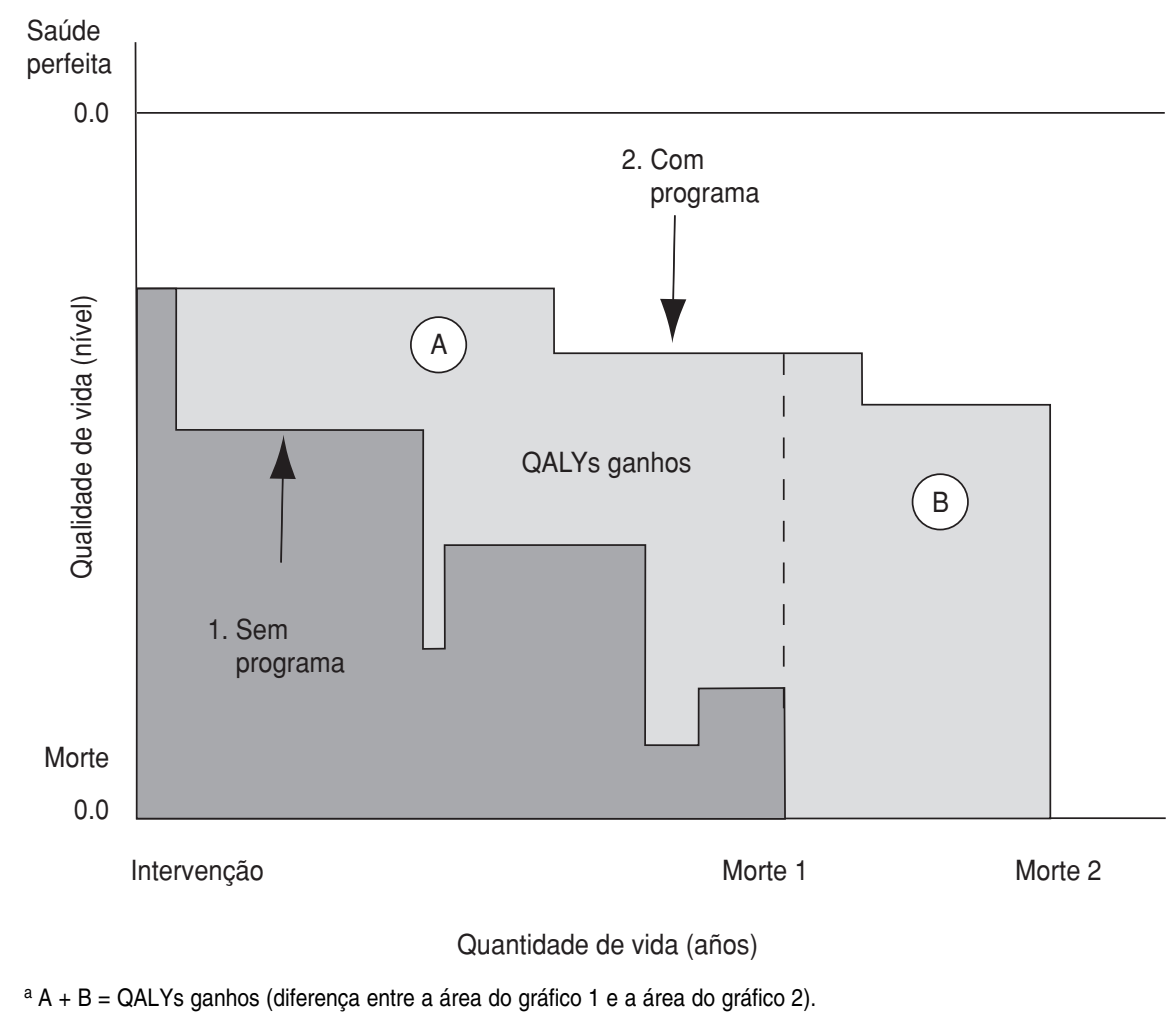

Como o modelo QALY pode ser utilizado para as análises de custo-utilidade em saúde, as medidas de utilidade são fundamentais para se determinar o peso das preferências por estados de saúde dentro desse modelo. A grande vantagem dessa abordagem é que ela estabelece uma medida padrão para comparar doenças e programas nas avaliações de incorporação de tecnologia em saúde (43).

\section{COMENTÁRIOS FINAIS}

Provavelmente, o século XXI será marcado por tentativas de integrar modelos biomédicos e psicossociais (44). Dentro de um modelo integrado de saúde, as contribuições do constructo qualidade de vida serão cada vez maiores, tanto da perspectiva da atenção individual quanto da coletiva (7).

Na perspectiva populacional, os instrumentos de avaliação de qualidade de vida devem permitir a vigilância epidemiológica, a avaliação do efeito das políticas públicas e a alocação de recursos conforme às necessidades de saúde. Já da perspectiva individual, esses instrumentos devem potencializar a avaliação diagnóstica da natureza e da severidade das doenças, traçar prognósticos, avaliar a eficácia terapêutica e levantar fatores etiológicos (7).

Dentre os instrumentos de avaliação de qualidade de vida, os índices de saúde (ou medidas de utilidade) compreendem ferramentas capazes de agregar em uma única medida vários dos propósitos descritos. Ao mesmo tempo, possibilitam a estruturação de análises de decisão clínica e análises econômicas em saúde, a construção de novos indicadores populacionais de saúde (QALYs) e novas descrições individuais de estados de saúde (sistemas multi-atributos).

Se estamos diante de um novo paradigma de saúde, só o tempo mostrará. Seja como for, há fortes indícios de um futuro em que o gerenciamento clínico será feito com base nas melhores evidências científicas disponíveis, em que a vigilância epidemiológica se baseará em indicadores integrados de morbi-mortalidade e em que a gestão em saúde levará em conta as opiniões e preferências dos usuários do sistema.

Apostar nesse paradigma é, antes de tudo, acreditar que a prática médica deve ser alicerçada na ciência, permeada pela arte, mas, sobretudo, compromissada com a vida. 


\section{SYNOPSIS}

\section{Quality of life and utility measures: clinical parameters for decision-making in health}

In recent decades, the international scientific community has become increasingly interested in the concept of quality of life. One of the most important implications of the focus on quality of life is a shift from "cure" to "a guarantee of a better life" as a health care goal, as well as the inclusion of individuals' preferences for certain health states in the decision- making process associated with treatments, diagnostic strategies, and health spending. This is especially important as the prevalence of chronic diseases increases as a result of the aging of the population. This piece describes the main concepts and applications related to this new health paradigm, including quality of life itself, utility measures, qualityadjusted life years (QALYs), and health decision analysis.

Key words: costs and cost analysis, patient satisfaction, decision support techniques.

\section{REFERÊNCIAS}

1. Guillemin F, Bombardier C, Beaton D. Cross-cultural adaptation of healthrelated quality of life measures: literature review and proposed guidelines. J Clin Epidemiol. 1993;46(12):1417-32.

2. Fitzpatrick R, Fletcher A, Gore $S$, Jones D, Spiegelhalter D, Cox D. Quality of life measures in health care. I: Applications and issues in assessment. BMJ. 1992;305 (6861):1074-7.

3. Carr AJ, Thompson PW, Kirwan JR. Quality of life measures. Br J Rheumatol. 1996;35(3):275-81

4. Paschoal SMP. Qualidade de vida na velhice. Em: Freitas EV, Py L, eds. Tratado de geriatria e gerontologia. Rio de Janeiro: Editora Guanabara Koogan; 2002. Pp. 79-84.

5. Litvak J. El envejecimiento de la población: un desafío que va más allá del año 2000. Bol Oficina Sanit Panam. 1999;109 (1):1-5.

6. Bowling A, Brazier J. Quality of life in social science and medicine. Soc Sci Med. 1995;41(10):1337-8.

7. Ebrahim S. Clinical and public health perspectives and applications of healthrelated quality of life measurement. Soc Sci Med. 1995;41(10):1383-94

8. Berzon RA, Mauskopf JA, Simeon GP. Choosing a health profile (descriptive) and/or a patient-preference (utility) measure for a clinical trial. Em: Spilker B, editor. Quality of life and pharmacoeconomics in clinical trials. Philadelphia: Lippincott-Raven Publishers; 1995. Pp. 375-9.

9. Zhan L. Quality of Life: Conceptual and measurement issues. J Adv Nurs. 1992; 17(7):795-800.

10. The WHOQOL GROUP. The World Health Organization Quality of Life Assessment (WHOQOL): Position paper from the world health organization. Soc Sci Med. 1995;41(10):1403-9.

11. Revicki DA. Relationship of pharmacoeconomics and health-related quality of life. Em: Spilker B, editor. Quality of life and pharmacoeconomics in clinical trials. Philadelphia: Lippincott-Raven Publishers; 1996. Pp. 1077-83.

12. Guyatt GH. A taxonomy of health status instruments. J Rheumatol. 1995;22(6): 1188-90.

13. Kirshner B, Guyatt G. A Methodological framework for assessing health indices. J Chronic Dis. 1985;38(1):27-36.

14. Guyatt GH, van Zaten SJOV, Feeney $\mathrm{DH}$, Patrick DL. Measuring quality of life in clinical trials: a taxonomy and review. CMAJ. 1989;140(12):1441-8.

15. Feeny DH, Torrance GW, Labelle R. Integrating economic evaluation and quality of life assessments. Em: Spilker B editor. Quality of life and pharmacoeconomics in clinical trials. Philadelphia: Lippincott-Raven Publishers; 1996. Pp. 85-95.

16. Torrance GW, Feeny DH. Utilities and quality-adjusted life years. Int J Technol Assess Health Care. 1989;5(4):559-75.

17. Torrance GW, Feeny D, Furlong W. Visual analog scales: Do they have a role in the measurement of preferences for health states? Med Decis Making. 2001; 21(4):329-34.

18. Torrance GW, Furlong W, Feeny D. Health utility estimation. Expert Rev Pharmacoeconomics Outcomes Res. 2002;2(2):99-108.

19. Cramer JA, Spilker B. Utilities and outcomes. Em: Quality of life and pharmacoeconomics. Philadelphia: LippincottRaven; 1998. Pp. 193-219.

20. Bennett KJ, Torrance GW. Measuring health state preferences and utilities: rating scale, time trade-off, and standard gamble techniques. Em: Spilker B, editor. Quality of life and pharmacoeconomics in clinical trials. Philadelphia: Lippincott-Raven Publishers; 1996. Pp. 253- 265.

21. Martin AJ, Lumley TS, Simes RJ. Incorporating trade-offs in quality of life assessment. Quality of life and pharmacoeconomics in clinical trials. Philadelphia: Lippincott-Raven Publishers; 1996. Pp. 403-12.
22. Kind P. The EuroQol Instrument: An index of health-related quality of life. Em: Spilker B, editor. Quality of life and pharmacoeconomics in clinical trials. Philadelphia: Lippincott-Raven Publishers; 1996. Pp. 191-201.

23. Kaplan RM, Anderson JP. The general health policy model: an integrated approach. Em: Spilker B, editor. Quality of life and pharmacoeconomics in clinical trials. Philadelphia: Lippincott-Raven Publishers; 1996. Pp. 309-22.

24. Feeny DH, Torrance GW, Furlong WJ. Health utilities index. Em: Spilker B, editor. Quality of life and pharmacoeconomics in clinical trials. Philadelphia: Lippincott-Raven Publishers; 1996. Pp. 239-50.

25. Brazier J, Usherwood T, Harper R, Thomas K. Deriving preference-based single index from the UK SF-36 health survey. J Clin Epidemiol. 1998;51(11): 1115-28.

26. Conner-Spady B, Suárez-Amazor ME. Variation in the estimation of qualityadjusted life-years by different preference-based instruments. Med Care. 2003;41(7):791-801.

27. Meletiche DM, Doshi D, Lofland JH. Medical outcomes study short-form 36: a possible source of utilities? Clin Ther. 1999;21(11):2016-26.

28. O'Brien BJ, Spath M, Blackhouse G, Severens JL, Dorian P, Brazier J. A view from the bridge: agreement between the SF-6D utility algorithm and the health utilities index. Health Econ. 2003;12(11): $975-81$.

29. Ciconelli RM, Ferraz MB, Santos W, Meinão I, Quaresma MR. Tradução para a língua portuguesa e validação do questionário genérico de avaliação de qualidade de vida SF-36 (Brasil SF-36). Rev Bras Reumatol. 1999;39(3):143-50.

30. McGhan WF. Using decision-analysis approaches to integrate QOL and cost data in drug therapy selection. Em: Spilker B, editor. Quality of life and pharmacoeconomics in clinical trials. 
Philadelphia: Lippincott-Raven Publishers; 1996. Pp. 741-5.

31. Sox HC. Decision Analysis: A basic clinical skill? N Engl J Med. 1987;316(5):271-2.

32. Kassirer JP, Moskowitz AJ, Lau J, Pauker SG. Decision analysis: a progress report. Ann Intern Med. 1987;106(2): 275-91.

33. Shlipak MG. Análise de decisão. Em: Friedland, editor. Medicina baseada em evidências: uma estrutura para a prática clínica. Rio de Janeiro: Editora Guanabara Koogan; 2001. Pp. 32-51.

34. Cramer JA, Spilker B. Pharmacoeconomics. Em: Quality of life and pharmacoeconomics. Philadelphia: LippincottRaven; 1998. Pp. 221-64.

35. Petitti DB. Decision analysis. Em: Petitti DB. Meta-analysis, decision analysis and cost-effectiveness analysis. New York: Oxford University Press; 2000. Pp. 17-28.

36. Rittenhouse B E. Designing and conducting cost-minimization and cost- effectiveness analyses. Em: Spilker B, editor. Quality of life and pharmacoeconomics in clinical trials. Philadelphia: Lippincott-Raven Publishers; 1996. Pp. 741-5.

37. Birch S, Gafni A. Cost effectiveness/ utility analyses. Do current decision rules lead us to where we want to be? J Health Econ. 1992;11(3):279-96.

38. O'Brien B. Principles of economic evaluation for health care programs. J Rheumatol. 1995;22(7):1399-402.

39. Torrance GW. Designing and conducting cost-utility analyses. Em: Spilker B, editor. Quality of life and pharmacoeconomics in clinical trials. Philadelphia: Lippincott-Raven Publishers; 1996. Pp. 1105-11.

40. Mulley AG. Assessing patient's utilities: can the ends justify the means? Med Care. 1989;27(3):S260-81.

41. Williams AH. Health policy implications of using quality of life measures in the economic evaluation of health care. Em: Spilker B, editor. Quality of life and pharmacoeconomics in clinical trials. Philadelphia: Lippincott-Raven Publishers; 1996. Pp. 753-9.

42. Minshall ME. Cost-utility analysis: the nirvana to health economists and an enigma to decision-makers? Expert Rev Pharmacoeconomics Outcomes Res. 2002;2(1):3-5.

43. Eddy DM. Rules for evaluating medical technologies. Em: Spilker B, editor. Quality of life and pharmacoeconomics in clinical trials. Philadelphia: LippincottRaven Publishers; 1996. Pp. 761-71.

44. Wilson IB, Kaplan S. Clinical practice and patients' health status: how are the two related? Med Care. 1995;33(Suppl): AS209-14.

\title{
International AIDS Conference
}

\author{
Dates: $\quad$ 13-19 August 2006 \\ Location: Metro Toronto Convention Centre \\ Toronto, Ontario, Canada
}

Twenty-five years after the first reports of AIDS, the magnitude of the epidemic demands increased accountability on the part of all stakeholders. With this in mind, the theme of the XVI International AIDS Conference is "Time to Deliver." That theme emphasizes that while research to expand and improve the understanding of HIV is ongoing, the scientific knowledge and tools to prevent new infections and prolong life already exist.

The conference will include a wide variety of plenary sessions and concurrent sessions as well as such other activities as a youth program, a cultural program, and a "global village." Many of the program sessions will deal with deal four key challenges in the fight against HIV/AIDS: accelerating research to end the epidemic, expanding and sustaining human resources to scale up prevention and treatment, intensifying the involvement of affected communities, and building new leadership to advance the response. Other meeting sessions will focus on lessons from the field related to scaling up, with special presentations, group discussions, and other activities that will highlight model programs and discuss key barriers to overcoming the current challenges.

For regular delegates from non-OECD countries, the registration fee for the conference is US\$ 660 until 15 May, and US\$ 730 after that date. For persons from OECD countries, the respective fees are US\$ 895 and US\$ 995. There are much lower fees for students, from both OECD and non-OECD countries. Some scholarship funds will be available for persons from developing countries, people living with HIV/AIDS, and young scientists.

\section{Information:}

International AIDS Society

P.0. Box 2

$\mathrm{CH}-1216$ Cointrin

Geneva, Switzerland

Telephone: $41-(0) 22-7100800$

Fax: 41-(0)22-7 100899

E-mail: info@iasociety.org

Web site: http://www.aids2006.org/contact.aspx 